\title{
CORRELATION RELATIONSHIPS FOR THE HYPOPLASTIC MODEL FOR FINE GRAINED SOILS
}

\author{
Tomáš KadlíčEK*， Tomáš Janda, Michal ŠEjnoha \\ Department of Mechanics, Faculty of Civil Engineering, Czech Technical University in Prague, \\ Thákurova 7/2077, 16629 Prague, Czech Republic \\ * corresponding author: tomas.kadlicek@fsv.cvut.cz
}

\begin{abstract}
.
The paper is concerned with our ongoing research effort devoted to the development of reliable computational tools for the calibration of advanced constitutive models of soils. At present, such software is available for the hypoplastic model of clays applicable to soft soils. This software provides a stepping stone for the determination of potential links between individual model parameters and fundamental characteristics of soils. Identifying such links would allow for tuning the model without performing time consuming experiments, particularly in the case of an initial design. Some preliminary results are presented in the paper.
\end{abstract}

KEYWORDS: hypoplasticity, fine grained soils, constitutive modeling, correlation.

\section{INTRODUCTION}

The theory of hypoplasticity has been receiving considerable attention in recent years, particularly for its capability of well simulating the basic characteristics of soils resulting in reliable predictions at a structural level. These characteristics include not only the dependency of stiffness on the void ratio, but also the stiffness dependency on strain and the loading history. Such relationships are hardly available with frequently used elastoplastic models of the Mohr-Coulomb (MC). These are generally adopted in FEM simulations because of their simplicity and straightforward links to basic strength parameters such cohesion and the angle of internal friction, which are included in regular laboratory test protocols or available in common material databases.

To provide a similar comfort or at least considerably simplify the use of hypoplastic models is the principal objective of our current research focusing particularly on the Masin hypoplastic model for fine grained soils (HC) [1] and the Von Wolffersdorff hypoplastic model for coarse grained soils (VW) [2]. In particular, two specific directions are investigated. The first aims at creating easy to use calibration software built upon standard oedometric and triaxial laboratory tests. The second option assumes a calibration procedure in the form of tables or graphs enabling the determination of model parameters on the basis of specific input data. Knowing the link between the model parameters and more standard soil parameters creates the basis for the development of such a tool. This objective is examined in this paper and some preliminary results are presented.

\section{Hypoplastic MODEL FOR FINE GRAINED SOILS}

The theory of hypoplasticity can be considered a possible alternative to the theory of elastoplasticity. Unlike elastoplasticity, it does not separate an elastic and plastic deformation, but its deformation is controlled by the evolution of state variables, namely pressure and the void ratio. Consequently, hypoplasticity exhibits a distinct nonlinear deformation both in primary loading and elastic unloading. The state variables are bound in the $e \times q \times p$ (e âĂŞ void ratio, q - deviatoric stress, $\mathrm{p}$ - mean stress) space, where all admissible states are enclosed by the so called state boundary surface (SBS).

The HC model defines five material parameters: $\lambda^{*}$, $\kappa^{*}, N, \varphi_{c}$ and $\nu$. These parameters can be calibrated on the basis of simple oedometric and triaxial laboratory tests. When properly tuned the model provides reliable and accurate predictions of the soil response in the solution of real engineering tasks.

The first three parameters: $\lambda^{*}, \kappa^{*}$ and $N$ are closely related to the oedometric test. As seen in Fig. 1 . the parameter $\lambda^{*}$ controls the slope of the Normal Consolidation Line (NCL). The position of NCL in the $\ln p \times \ln (e+1)$ space is driven by the parameter $N$. Clearly, recall Fig. 11 its value corresponds to the mean pressure $\mathrm{p}=1 \mathrm{kPa}$. The third parameter $\kappa^{*}$ represents the slope of the swelling line followed upon unloading as also evident from Fig. 1 For more details, the interested reader is referred to [1] and [2].

The remaining two parameters $\varphi_{c}$ and $\hat{I}_{j}$ are typically determined on the basis of the triaxial test. In the case of fine grained soils, an undrained triaxial test accompanied by measuring the pore pressure is preferred. The critical state friction angle $\varphi_{c}$ is dis- 


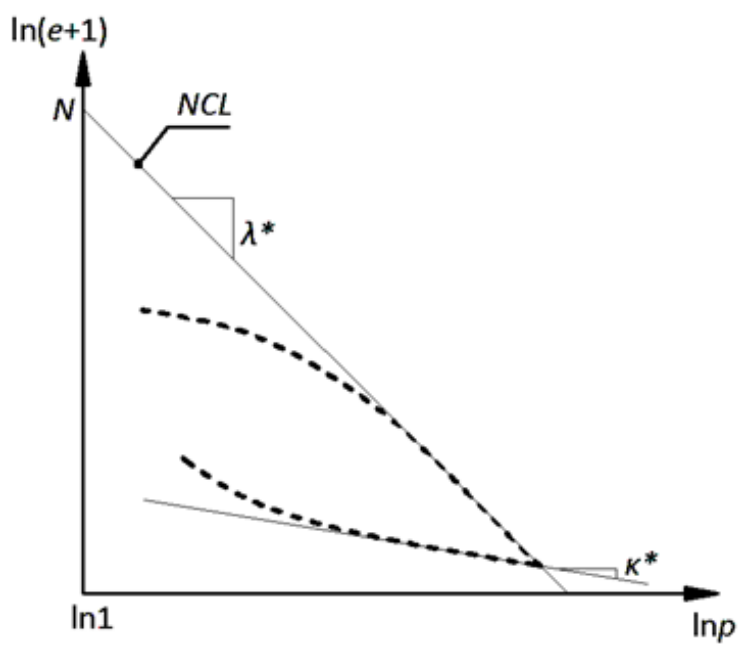

Figure 1. Parameters $\lambda^{*}, \kappa^{*}$ and $N$ in the $\ln p \times$ $\ln (e+1)$ space

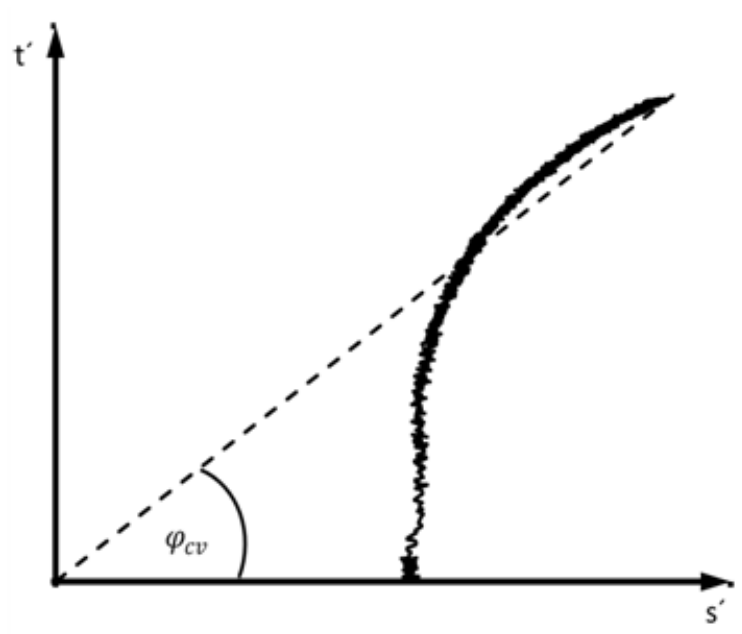

Figure 2. Critical friction angle $\varphi_{c}$ in the $t^{\prime} \times s^{\prime}$ space

played in Fig. 2. The effective stresses $t^{\prime}$ and $s^{\prime}$ in Fig. 2 are written as

$$
s^{\prime}=\frac{\sigma_{1}^{\prime}+\sigma_{3}^{\prime}}{2} \quad t^{\prime}=\frac{\sigma_{1}^{\prime}-\sigma_{3}^{\prime}}{3}
$$

The last parameter $\hat{\mathrm{I}}_{i}$ is related to the $K_{i} / G_{i}$ ratio, where $K_{i}$ is the bulk modulus in isotropic compression and $G_{i}$ is the shear modulus in undrained shear for a test starting from the isotropic normally compressed state. Obtaining a reliable prediction of this parameter requires a parametric study. The result of such a parametric study in terms of an equivalent deviatoric stress plotted as a function of the vertical strain is shown in Fig. 3. Additional details are available in 1] and [2].

Further details on the proposed calibration procedure for both $\mathrm{HC}$ and VW models, developed as well as validated on the basis of a large ensemble of laboratory tests, are provided in [3. In the next section, limiting our attention to the $\mathrm{HC}$ model, the model parameters determined for a representative set of soil samples will be correlated with a selected set of other, easily determined, soil characteristics.

\section{HC PARAMETERS' CORRELATIONS}

Soils can be characterized and classified according to the particle-size distribution and Atterberg limits. In order to determine the size distribution of particles in a specimen the sedimentation test (for grain sizes $<0.063 \mathrm{~mm}$ ) and measurements of the amount of particles passing a particular sieve size can be performed to derive the grain size distribution curve. The Atterberg limits are determined as the values of mass moisture content of a soft soil and are defined as follows:

- Shrinkage limit $W_{s}$

is the water content where an ongoing loss of moisture is not followed by any additional volume reduction

- Plastic limit $W_{p}$

is the water content where cylindrical samples of soil being $3 \mathrm{~mm}$ in diameter fall apart in pieces 8-10mm long

- Liquid limit $W_{l}$

is the water content where a groove made in soil placed in the Casagrande device merges at a length of $10 \mathrm{~mm}$ after 20 drops.

In the present study, the results from oedometric and triaxial measurements and index tests carried out on eight samples of soft soils were exploited. While the first set of experiments was employed in the calibration procedure to assess the values of individual model parameters, the actual soil classification was based on the index tests. The resulting values are listed in Tab. 1. Apart from model parameters and the Atterberg limits $W_{l}$ and $W_{p}$, the table also offers the percentage content of fine soil particles fwith the diameter $\mathrm{d}<$ $0.063 \mathrm{~mm}$. The particle-size distribution is also plotted in Fig. 4 for the sake of clarity.

When searching for possible correlations between the model parameters and the selected soil characteristics it has been proven convenient to adopt parameters calibrated on the basis of reconstituted soil samples. These samples were prepared from the original undisturbed soil by changing the sample paste consistency up to a water content of $1.5 \times W_{l}$ while completely disrupting their original internal structure and as such losing any information about the prior loading history. The values of model parameters are nevertheless the same as those which would have been found when adopting the original undisturbed samples.

The relationships between the values of $\mathrm{HC}$ parameters and the index characteristics, see Fig. 5 and 6 , can be conveniently specified in terms of the coefficient of correlation $r \in(-1 ; 1)$. Recall that the correlation reflects the noisiness and direction of a linear relationship of two random variables, but not the slope of that relationship. When there is no obvious relationship 


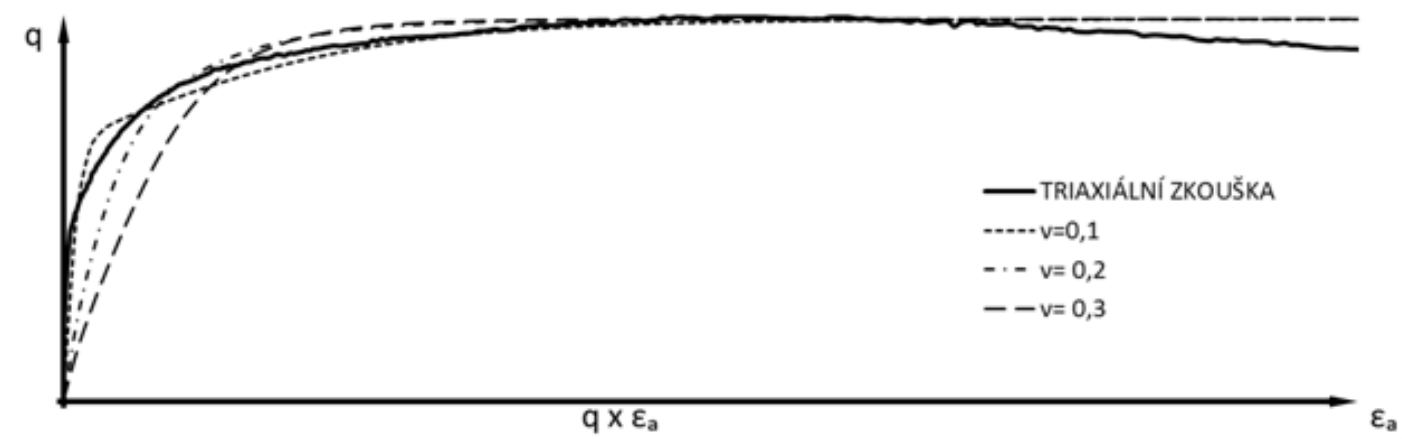

Figure 3. Evolution of the equivalent deviatoric stress $q$ as a function of the vertical strain $\varepsilon_{a}$ for various values of $\nu$

\begin{tabular}{|l|c|c|c|c|c|c|c|c|c|}
\hline \multirow{2}{*}{ Specimen } & \multirow{2}{*}{ USCS } & \multicolumn{7}{|c|}{$\mathrm{HC}$} & \multicolumn{3}{|c|}{} \\
\cline { 3 - 10 } & & $\lambda^{*}$ & $\kappa^{*}$ & $N$ & $\varphi_{C S}$ & $v$ & $\mathrm{~W}_{1}$ & $\mathrm{~W}_{\mathrm{p}}$ & $\mathrm{f}$ \\
\hline Prackovice 1 & $\mathrm{CH}$ & 0,060 & 0,018 & 1,029 & 29 & 0,202 & 66,7 & 30,8 & 97,4 \\
\hline Prackovice 2 & $\mathrm{CH}$ & 0,065 & 0,018 & 1,069 & 29 & 0,185 & 66,7 & 30,8 & 97,4 \\
\hline Praha A & $\mathrm{CL}$ & 0,053 & 0,013 & 0,720 & 31 & 0,002 & 34,8 & 20 & 64,9 \\
\hline Ujezd & $\mathrm{Cl}$ & 0,067 & 0,008 & 0,940 & 31 & 0,444 & 35 & 21 & 87,6 \\
\hline Bilina 1 & $\mathrm{CL}$ & 0,043 & 0,011 & 0,698 & 25 & 0,299 & 49,7 & 26,9 & 64,6 \\
\hline Bilina 2 & $\mathrm{CH}$ & 0,055 & 0,013 & 0,954 & 23 & 0,321 & 59,9 & 25,5 & 92,2 \\
\hline Hajek & $\mathrm{CL}$ & 0,035 & 0,007 & 0,606 & 33 & 0,111 & 48,5 & 22,6 & 57 \\
\hline Zaječí & $\mathrm{CH}$ & 0,068 & 0,022 & 1,078 & 27 & 0,018 & 75 & 34,7 & 91,1 \\
\hline
\end{tabular}

TABle 1. Parameters of the HC model and selected parameters from index tests for reconstituted soil samples

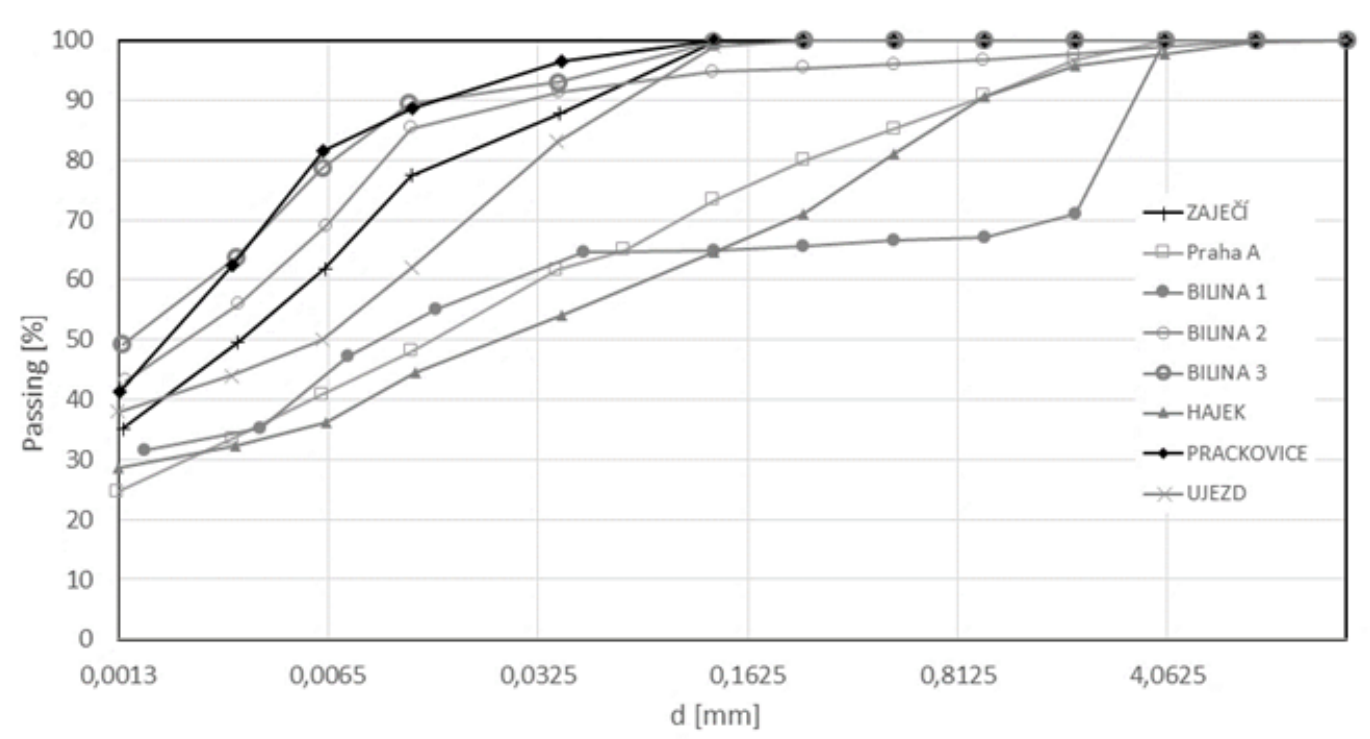

Figure 4. Particle-size distribution for eight samples of sampled soils 


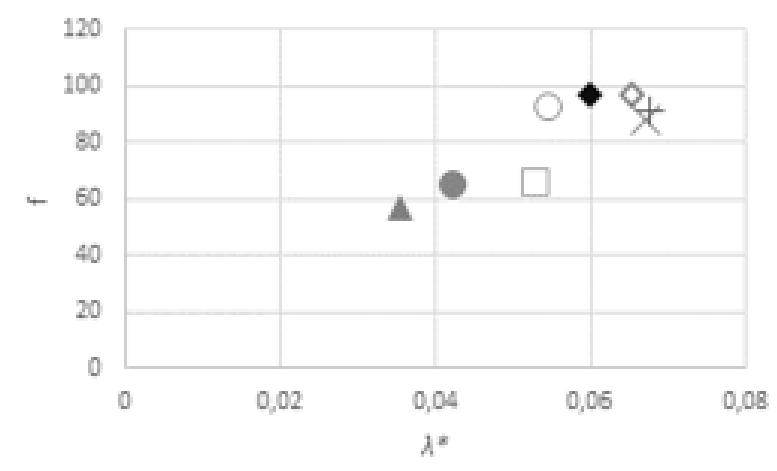

a)

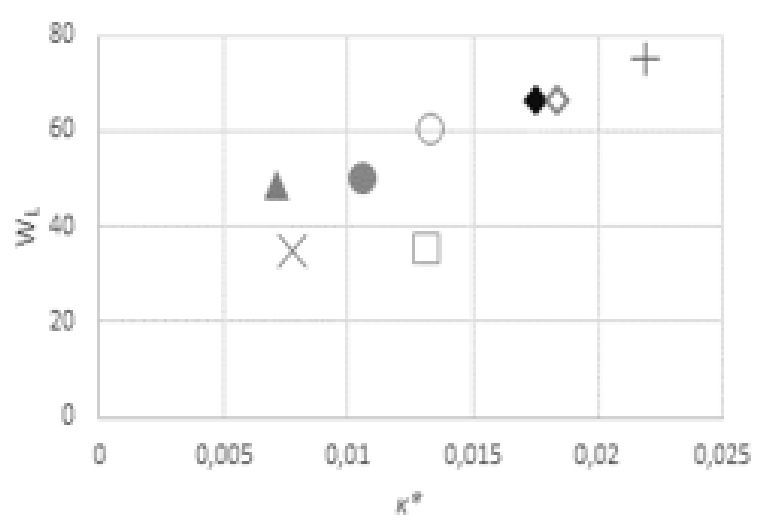

c)

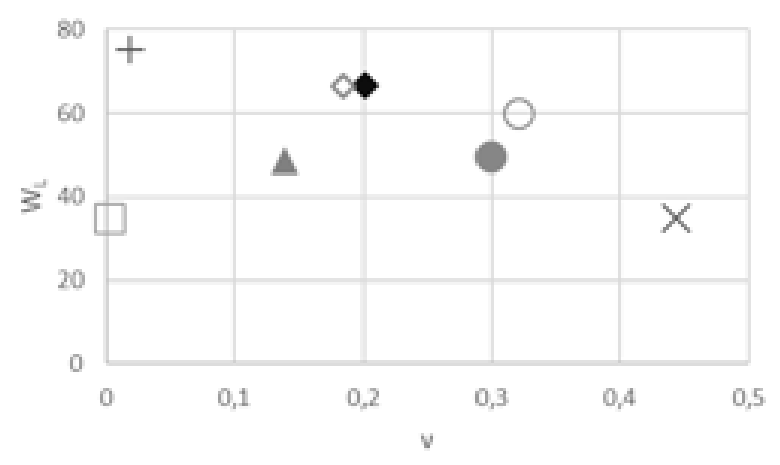

e)

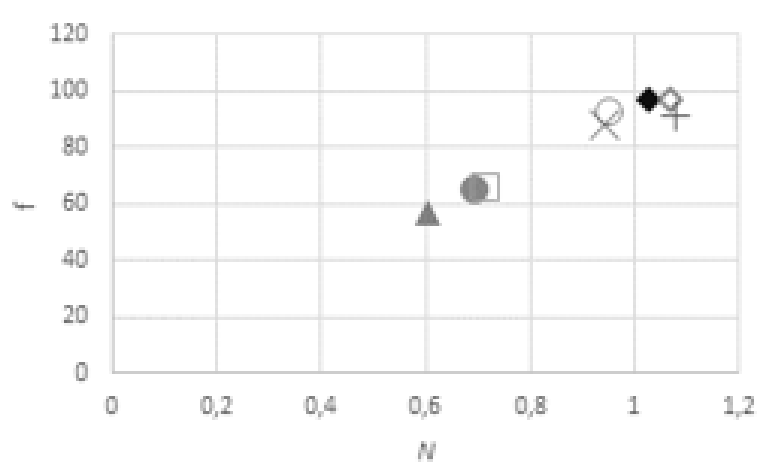

b)

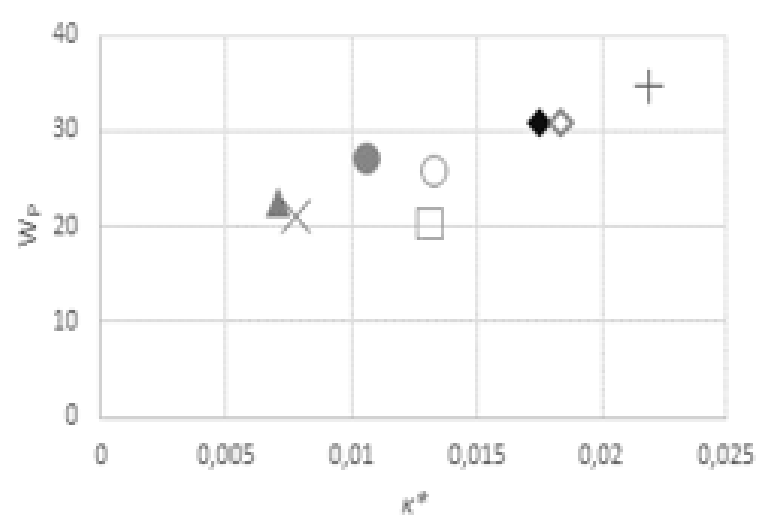

d)

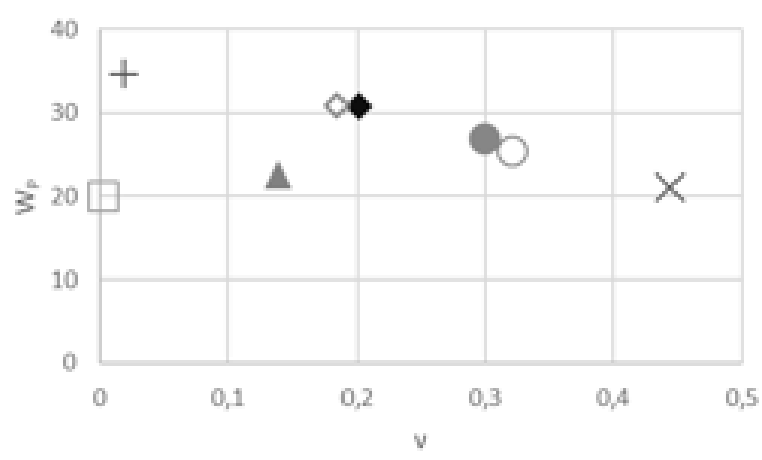

$f$

Figure 5. Correlations between HC parameters and index characteristics

(correlation), the coefficient of correlation is equal to 0 . In the case of a perfect direct (increasing) correlation, $r$ approaches 1 and in the case of a perfect inverse (decreasing) correlation, $r$ approaches -1 . Thus, the closer the coefficient is either to -1 or 1 , the stronger the correlation between the two variables.

Tab. 2 shows the coefficient of correlation for all five $\mathrm{HC}$ model parameters and the selected index characteristics $W_{l}$ and $W_{p}$, and the percentage content of fine soil particles $f$. A relatively strong (direct) correlation is observed for the model parameters $\lambda^{*}$ and $N$ and the content of fine soil particles $f$. This is also confirmed by the plots in Fig. 5(a,b) showing no apparent scattering. Remember that the parameters $\lambda^{*}$ and $N$ control the position of NCL in the $\ln p \times$ $\ln (e+1)$ space. Both Tab. 2 and the plots in Fig. $5(\mathrm{c}, \mathrm{d})$ suggest the parameter $\kappa^{*}$ be more dependent on the plasticity index $W_{p}$ and the liquid limit $W_{l}$. The same conclusion, although not directly visible from Tab. 2 can be drawn for the parameter $v$, see Fig. $5(\mathrm{e}, \mathrm{f})$. For this parameter, the value of the coefficient of correlation is polluted by the results derived from the soil sample labeled as "Praha A", which falls off the more or less linear relationship. This specimen 


\begin{tabular}{|c|c|c|}
\hline \multicolumn{3}{|c|}{$\lambda^{*}$} \\
\hline$W I$ & $W p$ & $f$ \\
\hline \multicolumn{3}{|c|}{$r$} \\
\hline 0,35 & 0,43 & 0,85 \\
\hline
\end{tabular}

\begin{tabular}{|c|c|c|}
\hline \multicolumn{3}{|c|}{$N$} \\
\hline$W I$ & $W p$ & $f$ \\
\hline \multicolumn{3}{|c|}{$r$} \\
\hline 0,67 & 0,69 & 0,98 \\
\hline
\end{tabular}
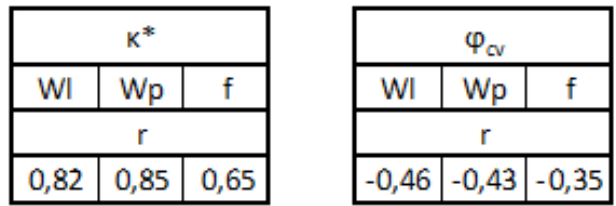

\begin{tabular}{|c|c|c|}
\hline \multicolumn{3}{|c|}{$v$} \\
\hline$W I$ & $W p$ & $f$ \\
\hline \multicolumn{3}{|c|}{$r$} \\
\hline 0,72 & 0,71 & 0,58 \\
\hline
\end{tabular}

TABLE 2. Correlation values $r$

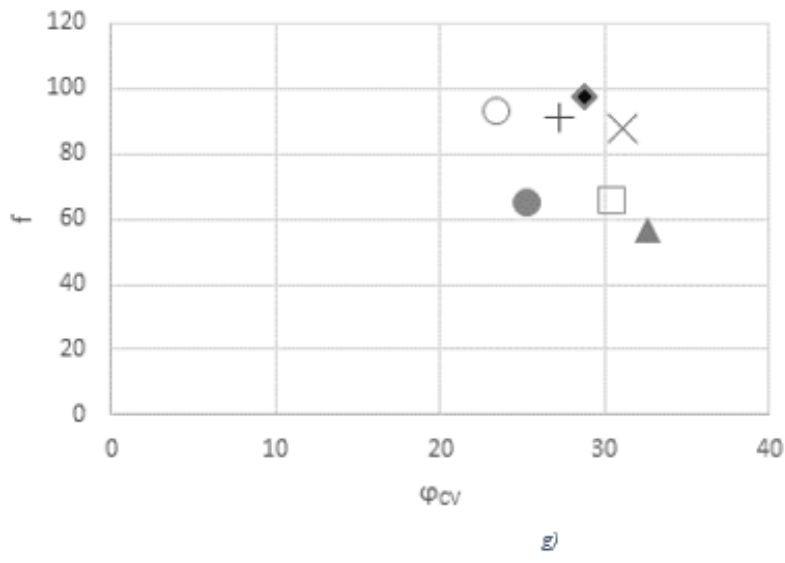

Figure 6. Correlations between $\mathrm{HC}$ parameters and index characteristics

shows the lowest values of the Atterberg limits and the lowest content of fine soil particles $f$. Unlike the first four parameters associated more or less with the compressive response of soil, the critical state friction angle $\varphi_{c v}$ does not seem to show any correlation with the selected index characteristics, see both Tab. 2 and Fig. 6(g). However, its determination is rather simple from a standard triaxial test with no need for additional optimization.

\section{Conclusion}

Although drawing a solid conclusion as to the direct link of the HC model parameters to other, more standard, soil parameters would require a considerably larger set of experiments, the potential of this goal has been clearly confirmed by this study. In particular, a relatively strong correlation of the $\mathrm{HC}$ model parameters $\lambda^{*}, \kappa^{*}, \mathrm{~N}$ and $\nu$ and the selected index parameters or the particle size distribution data can be found.

While limiting our attention to the $\mathrm{HC}$ model we expect that a similar study performed for the VW model can lead to similar conclusions. Obviously, this must be supported by a much larger set of data to be examined. We expect such information to grow with the help of a web application which not only provides calibration software, but opens a way to collected results from laboratory measurements all over the world.

\section{ACKNOWLEDGEMENT}

The financial support from projects TA04031603 and SGS16/038/OHK1/1T/11 is gratefully acknowledged.

\section{REFERENCES}

[1] D. Mašín. Clay hypoplasticity with explicitly defined asymptotic states. Acta Geotechnica 8(5):481-496, 2013. DOI:10.1007/s11440-012-0199-y.

[2] D. Mašín. A hypoplastic constitutive model for clays. International Journal for Numerical and Analytical Methods in Geomechanics 29(4):311-336, 2005. DOI:10.1002/nag.416.

[3] M. Šejnoha, T. Kadlíček, T. Janda. Calibration of hypoplastic models for soils. In Engineering Mechanics 2015, vol. 821 of Applied Mechanics and Materials, pp. 503-511. Trans Tech Publications, 2016. DOI:10.4028/www.scientific.net/AMM.821.503

[4] P. A. von Wolffersdorff. A hypoplastic relation for granular materials with a predefined limit state surface. Mechanics of Cohesive-frictional Materials 1(3):251-271, 1996. DOI:10.1002/(SICI)10991484(199607)1:3<251::AID-CFM13>3.0.CO;2-3 